\title{
The parametrix method for parabolic SPDEs
}

\author{
Andrea Pascucci* $\quad$ Antonello Pesce ${ }^{\dagger}$
}

This version: December 13, 2019

\begin{abstract}
We consider the Cauchy problem for a linear stochastic partial differential equation. By extending the parametrix method for PDEs whose coefficients are only measurable with respect to the time variable, we prove existence, regularity in Hölder classes and estimates from above and below of the fundamental solution. This result is applied to SPDEs by means of the Itô-Wentzell formula, through a random change of variables which transforms the SPDE into a PDE with random coefficients.
\end{abstract}

Keywords: stochastic partial differential equations, fundamental solution, parametrix method, Kolmogorov equation

\section{Introduction}

Stochastic partial differential equations (SPDEs) arise in many applications in probability theory and in particular in the study of filtering problems (see e.g. [8, [15]). Assume that $\left(X_{t}, Y_{t}\right)$ is a diffusion where $X$ represents a signal that is not directly observable and we want to extract information about $X$ from $\mathcal{F}_{t}^{Y}=\sigma\left(Y_{s}, s \leq t\right)$ that is the filtration of the observations on $Y$. Then, under natural assumptions, for any bounded and measurable function $f$ we have

$$
E\left[f\left(X_{t}\right) \mid \mathcal{F}_{t}^{Y}\right]=\int_{\mathbb{R}^{d}} f(x) p_{t}(x) d x
$$

where $p_{t}(x)$ denotes the conditional density of $X_{t}$ given $\mathcal{F}_{t}^{Y}$ : it turns out that $p_{t}$ satisfies a SPDE of the form

$$
d p_{t}(x)=\mathbf{L}_{t} p_{t}(x) d t+\mathbf{G}_{t} p_{t}(x) d W_{t},
$$

where $\mathbf{L}_{t}$ is a second-order elliptic operator and $\mathbf{G}_{t}$ is a first-order operator. The coefficients of $\mathbf{L}_{t}$ and $\mathbf{G}_{t}$ may depend on $t, x, Y_{t}$ and are therefore random and typically not smooth. A very particular case is when $Y \equiv 0$ : then $\mathbf{G}_{t}=0$ and (1.1) reduces to the classical forward Kolmogorov equation for the transition density $p_{t}$ of $X_{t}$. In the general case, $p_{t}$ can be referred to as the stochastic fundamental solution of (1.1). The aim of this paper is to prove existence, regularity and estimates from above and below of $p_{t}$ by using a classical tool from PDEs' theory, the parametrix method.

Let $(\Omega, \mathcal{F}, P)$ be a complete probability space with an increasing filtration $\left(\mathcal{F}_{t}\right)_{t \geq 0}$ of complete with respect to $(\mathcal{F}, P) \sigma$-fields $\mathcal{F}_{t} \subseteq \mathcal{F}$. Let $d_{1} \in \mathbb{N}$ and let $W^{k}, k=1, \cdots, d_{1}$, be one-dimensional independent Wiener processes with respect to $\left(\mathcal{F}_{t}\right)_{t \geq 0}$. We consider the parabolic SPDE

$$
d u_{t}(x)=\left(\mathbf{L}_{t} u_{t}(x)+\mathbf{f}_{t}(x)\right) d t+\mathbf{G}_{\sigma_{t}^{k}} u_{t}(x) d W_{t}^{k},
$$

\footnotetext{
*(Corresponding author) Dipartimento di Matematica, Università di Bologna, Bologna, Italy. e-mail: andrea.pascucci@unibo.it

†Dipartimento di Matematica, Università di Bologna, Bologna, Italy. e-mail: antonello.pesce2@unibo.it
} 
where $\mathbf{L}_{t}$ is the second-order operator

$$
\mathbf{L}_{t} u_{t}(x)=\frac{1}{2} \mathbf{a}_{t}^{i j}(x) \partial_{i j} u_{t}(x)+\mathbf{b}_{t}^{j}(x) \partial_{j} u_{t}(x)+\mathbf{c}_{t}(x) u_{t}(x)
$$

and $\mathbf{G}_{\sigma_{t}^{k}}$ is the first-order operator

$$
\mathbf{G}_{\sigma_{t}^{k}} u_{t}(x)=\sigma_{t}^{i k}(x) \partial_{i} u_{t}(x)
$$

Throughout the paper, the summation convention over repeated indices is enforced regardless of whether they stand at the same level or at different ones. The point of $\mathbb{R}^{d}$ is denoted by $x=\left(x_{1}, \ldots, x_{d}\right)$ and we set $\partial_{i}=\partial_{x_{i}}, \nabla=\left(\partial_{1}, \ldots, \partial_{d}\right)$ and $\partial_{i j}=\partial_{i} \partial_{j}$. A function $u=u_{t}(x, \omega)$ on $[0, \infty) \times \mathbb{R}^{d} \times \Omega$ is denoted by $u_{t}(x)$ and we shall systematically omit the explicit dependence on $\omega \in \Omega$. The coefficients $\mathbf{a}_{t}, \mathbf{b}_{t}$, $\mathbf{c}_{t}, \mathbf{f}_{t}$ and $\sigma_{t}^{k}$ in (1.2) are intended to be random and not smooth.

The Cauchy problem for evolution SPDEs has been studied by several authors. Under coercivity conditions analogous to uniform ellipticity for PDEs, there exists a complete theory in Sobolev spaces (see [20, [16, 25, 6] and references therein) and in the spaces of Bessel potentials (see [11] and [12]). Classical solutions in Hölder classes were first considered in [24, 26] and more recent results were proved in [1] and [18, though the authors only considered equations with non-random coefficients and with no derivatives of the unknown function in the stochastic term.

In the last decades, the use of analytical or PDE techniques in the study of SPDEs has become widespread. For instance, the results in [1, [18, [30] are based on classical methods of deterministic PDEs, such as Duhamel principle and a priori Schauder estimates; the $L^{p}$ estimates in 3 are proved by adapting the classical Moser's iterative argument; 28, provides short-time asymptotics of random heat kernels. A further remarkable example is given by the recent series of papers by Krylov [13, 14, 15] where the Hörmander's theorem for SPDEs is proved; see also the very recent results in 23 for backward SPDEs.

In this paper we extend another classical tool that, to the best of our knowledge, has not yet been considered in the study of SPDEs, the well-known parametrix method for the construction of the fundamental solution of PDEs with Hölder continuous coefficients. There are two main problems that one faces when trying to apply the parametrix method to SPDEs: the lack of a Duhamel principle for SPDEs and the roughness of the coefficients, that are assumed to be only measurable in time. In the first part of the paper, we use the Itô-Wentzell formula [29] to make a random change of variables which transforms the SPDE in a PDE with random coefficients; the latter admits a Duhamel principle and, in the second part of the paper, we use it to extend the parametrix method to parabolic PDEs with coefficients measurable in the time variable.

This paper does not pretend to encompass the most general assumptions but rather investigate the possible use of the parametrix method in the stochastic framework; as such, it has to be intended as a first step of a research programme aiming at considering more general classes of possibly degenerate SPDEs. More precisely, it is very likely that the techniques used in this paper can be applied to SPDEs satisfying the strong Hörmander condition, such as those considered in [13]. A more challenging problem is to consider the Langevin SPDE

$$
d u_{t}(x, y)=\left(\frac{a_{t}(x, y)}{2} \partial_{x x} u_{t}(x, y)+x \partial_{y} u_{t}(x, y)\right) d t+\sigma_{t}(x, y) \partial_{x} u_{t}(x, y) d W_{t}, \quad(x, y) \in \mathbb{R}^{2} .
$$

This equation has unbounded drift coefficient and satisfies the weak Hörmander condition. The parametrix method has been generalized to deterministic (i.e. with $\sigma_{t} \equiv 0$ ) Langevin PDEs in 22, 4] and 
10]; however, contrary to the uniformly parabolic case considered in the present paper, the intrinsic Hölder regularity in the spatial variables cannot be studied independently from the time variable as it was recently shown in [19]. This is an additional issue that needs careful investigation and is the subject of the forthcoming paper [21].

The paper is organized as follows. In Section 2 we introduce the basic notations and state our main result, Theorem 2.5. for illustrative purposes, the particular case of the stochastic heat equation is discussed in Subsection 2.1. In Section 3 we recall the Itô-Wentzell formula and provide some estimate for the related flow of diffeomorphisms. In Section 4 we present the parametrix method. Since the complete proofs are rather technical and to a large extent similar to the classical case, we only provide the details on those aspects that require significant modifications: in particular, in Section 4.3 we present a proof of the Gaussian lower bound for the fundamental solution which requires some non trivial adaptation of an original argument by Aronson (cf. [5]).

\section{Assumptions and main results}

Before stating our main theorems, we need to introduce some basic definitions and notations to be used throughout the paper. Let $k \in \mathbb{N}, \alpha \in(0,1)$ and $0 \leq t<T$. Denote:

- $C_{t, T}^{0}=C_{t, T}^{0}\left(\mathbb{R}^{d}\right)$ the space of all measurable functions $f=f_{s}(x)$ on $[t, T] \times \mathbb{R}^{d}$ that are continuous in $x$;

- $C_{t, T}^{\alpha}=C_{t, T}^{\alpha}\left(\mathbb{R}^{d}\right)$ the space of functions $f \in C_{t, T}^{0}$ that are $\alpha$-Hölder continuous in $x$ uniformly with respect to $s$, that is

$$
\sup _{\substack{s \in t, T] \\ s \neq y}} \frac{\left|f_{s}(x)-f_{s}(y)\right|}{|x-y|^{\alpha}}<\infty ;
$$

- $C_{t, T, \text { loc }}^{\alpha}$ the space of functions $f \in C_{t, T}^{0}$ such that

$$
\sup _{\substack{s \in[t, T] \\ x, y \in K, x \neq y}} \frac{\left|f_{s}(x)-f_{s}(y)\right|}{|x-y|^{\alpha}}<\infty
$$

for every compact subset $K$ of $\mathbb{R}^{d}$;

- $C_{t, T}^{k}$ the space of all measurable functions $f=f_{s}(x)$ on $[t, T] \times \mathbb{R}^{d}$ that are $k$-times differentiable w.r.t. $x$ with $\partial^{\beta} f \in C_{t, T}^{0}$ for any multi-index $\beta$ of height $|\beta|=k$;

- $C_{t, T}^{k+\alpha}$ (resp. $C_{t, T, \text { loc }}^{k+\alpha}$ ) the space of functions $f \in C_{t, T}^{k}$ with $\partial^{\beta} f \in C_{t, T}^{\alpha}$ (resp. $\partial^{\beta} f \in C_{t, T, \text { loc }}^{\alpha}$ ) for any multi-index $\beta$ of height $|\beta| \leq k$.

We use boldface to denote the stochastic versions of the previous functional spaces. More precisely, let now $k \in \mathbb{N} \cup\{0\}, \alpha \in[0,1)$ and $\mathcal{P}_{t, T}$ be the predictable $\sigma$-algebra on $[t, T] \times \Omega$. We denote by $\mathbf{C}_{t, T}^{k+\alpha}$ the family of functions $f=f_{s}(x, \omega)$ on $[t, T] \times \mathbb{R}^{d} \times \Omega$ such that:

i) $(s, x) \mapsto f_{s}(x, \omega) \in C_{t, T}^{k+\alpha} P$-a.s.;

ii) $(s, \omega) \mapsto f_{s}(x, \omega)$ is $\mathcal{P}_{t, T}$-measurable for any $x \in \mathbb{R}^{d}$.

Moreover, $\mathbf{b C}_{t, T}^{k+\alpha}$ is the space of functions $f \in \mathbf{C}_{t, T}^{k+\alpha}$ such that

$$
\sum_{|\beta| \leq k} \sup _{\substack{s \in[t, T] \\ x \in \mathbb{R}^{d}}}\left|\partial^{\beta} f_{s}(x)\right|<\infty \quad P \text {-a.s. }
$$


We say that $f=f_{s}(x)$ is non-rapidly increasing uniformly on $(t, T] \times \mathbb{R}^{d}$ if, for any $\delta>0, e^{-\delta|x|^{2}}\left|f_{s}(x)\right|$ is a bounded function on $(t, T] \times \mathbb{R}^{d}, P$-a.s.; in case $f$ does not depend on $s$, we simply say that $f$ is non-rapidly increasing on $\mathbb{R}^{d}$.

Definition 2.1. A stochastic fundamental solution $\boldsymbol{\Gamma}=\boldsymbol{\Gamma}(t, x ; \tau, \xi)$ for the SPDE (1.2) is a function defined for $0 \leq \tau<t \leq T$ and $x, \xi \in \mathbb{R}^{d}$, such that for any $(\tau, \xi) \in[0, T) \times \mathbb{R}^{d}$ we have:

i) $\boldsymbol{\Gamma}(\cdot, \cdot ; \tau, \xi) \in \mathbf{C}_{t_{0}, T}^{2}\left(\mathbb{R}^{d}\right)$ and with probability one satisfies

$$
\boldsymbol{\Gamma}(t, x ; \tau, \xi)=\boldsymbol{\Gamma}\left(t_{0}, x ; \tau, \xi\right)+\int_{t_{0}}^{t} \mathbf{L}_{s} \boldsymbol{\Gamma}(s, x ; \tau, \xi) d s+\int_{t_{0}}^{t} \mathbf{G}_{\sigma_{s}^{k}} \boldsymbol{\Gamma}(s, x ; \tau, \xi) d W_{s}^{k}
$$

for $\tau<t_{0} \leq t \leq T$ and $x \in \mathbb{R}^{d} ;$

ii) for any continuous and non-rapidly increasing function $\varphi$ on $\mathbb{R}^{d}$

$$
\lim _{\substack{(t, x) \rightarrow(\tau, \xi) \\ t>\tau}} \int_{\mathbb{R}^{d}} \boldsymbol{\Gamma}(t, x ; \tau, y) \varphi(y) d y=\varphi(\xi), \quad P \text {-a.s. }
$$

Next we state the standing assumptions on the coefficients of the SPDE (1.2).

Assumption 2.2 (Coercivity). Let

$$
\mathbf{A}_{t}(x):=\left(\mathbf{a}_{t}^{i j}(x)-\sigma_{t}^{i k}(x) \sigma_{t}^{j k}(x)\right)_{i, j=1, \ldots, d} .
$$

There exists a positive random variable $\lambda$ such that

$$
\left\langle\mathbf{A}_{t}(x) \xi, \xi\right\rangle \geq \lambda|\xi|^{2}, \quad t \in[0, T], x, \xi \in \mathbb{R}^{d}, P \text {-a.s. }
$$

Assumption 2.3 (Regularity). For some $\alpha \in(0,1)$ and for every $i, j=1, \ldots, d$ and $k=1, \ldots, d_{1}$, we have

$$
\mathbf{a}^{i j}, \mathbf{b}^{j}, \mathbf{c} \in \mathbf{b} \mathbf{C}_{0, T}^{\alpha} \quad \text { and } \quad \sigma^{i k} \in \mathbf{b C}_{0, T}^{3+\alpha} .
$$

We now introduce a random change of coordinates that will play a central role in the following analysis. We fix $(\tau, x) \in[0, T) \times \mathbb{R}^{d}$ and consider the stochastic ordinary differential equation

$$
x_{t}=x-\int_{\tau}^{t} \sigma_{s}^{k}\left(x_{s}\right) d W_{s}^{k}, \quad t \in[\tau, T] .
$$

It is well-known (see, for instance, Theor. 4.6.5 in [17])) that, under Assumption 2.3, equation (2.2) admits a solution $X=X_{\tau, t}(x, \omega)$ that is a stochastic flow of diffeomorphisms: precisely, $X_{\tau, t} \in \mathbf{C}_{\tau, T}^{3+\alpha^{\prime}}$, for any $\alpha^{\prime}<\alpha$, the matrix $\nabla X_{\tau, t}(x)$ satisfies

$$
\nabla X_{\tau, t}(x)=I_{d}-\int_{\tau}^{t} \nabla \sigma_{s}^{k}\left(X_{\tau, s}(x)\right) \nabla X_{\tau, s}(x) d W_{s}^{k}
$$

and, for any $i, j=1, \ldots, d, \partial_{i j}^{2} X_{\tau, t}(x)$ satisfies

$$
\partial_{i j}^{2} X_{\tau, t}^{h}(x)=-\int_{\tau}^{t}\left(\left(\nabla \sigma_{s}^{k}\left(X_{\tau, s}(x)\right) \partial_{i j}^{2} X_{\tau, s}(x)\right)_{h}+\left(\left(\nabla X_{\tau, s}(x)\right)^{*} \nabla^{2} \sigma_{s}^{h k}\left(X_{\tau, s}(x)\right) \nabla X_{\tau, s}(x)\right)_{i j}\right) d W_{s}^{k}
$$

with probability one.

Since we are going to use $X$ as a global change of variables, we need some control over the stochastic integrals in (2.3) and (2.4) for $x$ varying in $\mathbb{R}^{d}$ : this issue is addressed in Section 3 (see, in particular, Proposition 3.2) under the following additional condition. 
Assumption 2.4. There exist $\varepsilon>0$ and a random variable $M \in L^{\bar{p}}(\Omega)$, with $\bar{p}>\max \left\{2, d, \frac{d}{2 \varepsilon}\right\}$, such that

$$
\sup _{\substack{s \in[0, T] \\ x \in \mathbb{R}^{d}}}\left(1+|x|^{2}\right)^{\varepsilon}\left|\partial^{\beta} \sigma_{s}^{k}(x)\right| \leq M \quad P \text {-a.s. }
$$

for every $k=1, \cdots, d_{1}$ and multi-index $\beta$ with $1 \leq|\beta| \leq 3$.

Assumption 2.4 is a rather weak condition on the first, second and third order derivatives of $\sigma$ : clearly, it is satisfied under the very particular cases of $\sigma$ constant or $\sigma$ with compact support.

Our main result is the following

Theorem 2.5. Let Assumptions 2.2, 2.3 and 2.4 be in force. Then there exists a fundamental solution $\boldsymbol{\Gamma}$ for the SPDE (1.2). Moreover, there exist two positive random variables $\mu_{1}$ and $\mu_{2}$ such that

$$
\begin{aligned}
\frac{1}{\mu_{2}} \Gamma^{\frac{1}{\mu_{1}}}\left(t-\tau, X_{\tau, t}^{-1}(x)-\xi\right) & \leq \boldsymbol{\Gamma}(t, x ; \tau, \xi) \leq \mu_{2} \Gamma^{\mu_{1}}\left(t-\tau, X_{\tau, t}^{-1}(x)-\xi\right), \\
\left|\partial_{x_{i}} \boldsymbol{\Gamma}(t, x ; \tau, \xi)\right| & \leq \frac{\mu_{2}}{\sqrt{t-\tau}} \Gamma^{\mu_{1}}\left(t-\tau, X_{\tau, t}^{-1}(x)-\xi\right), \\
\left|\partial_{x_{i} x_{j}} \boldsymbol{\Gamma}(t, x ; \tau, \xi)\right| & \leq \frac{\mu_{2}}{t-\tau} \Gamma^{\mu_{1}}\left(t-\tau, X_{\tau, t}^{-1}(x)-\xi\right),
\end{aligned}
$$

for every $0 \leq \tau<t \leq T$ and $x, \xi \in \mathbb{R}^{d}$, where $\Gamma^{\mu}$ denotes the fundamental solution of the heat equation $\partial_{t} u_{t}(x)=\frac{\mu}{2} \Delta u_{t}(x)$.

The proof of Theorem 2.5 is postponed to Section 4.4

Corollary 2.6. Let $u_{0}$ be a $\mathcal{F}_{0} \otimes \mathcal{B}$-measurable function on $\Omega \times \mathbb{R}^{d}$ such that $u_{0}(\omega, \cdot)$ is continuous and non-rapidly increasing on $\mathbb{R}^{d}$ for a.e. $\omega \in \Omega$. Let $f \in \mathbf{C}_{0, T, \text { loc }}^{\bar{\alpha}}$, for some $\bar{\alpha} \in(0,1)$, be non-rapidly increasing uniformly on $[0, T] \times \mathbb{R}^{d}$. Then

$$
u_{t}(x)=\int_{\mathbb{R}^{d}} \boldsymbol{\Gamma}(t, x ; 0, \xi) u_{0}(\xi) d \xi+\int_{0}^{t} \int_{\mathbb{R}^{d}} \boldsymbol{\Gamma}(t, x ; s, \xi) \mathbf{f}_{s}(\xi) d \xi d s
$$

is a classical solution of (1.2) with initial value $u_{0}$, in the sense that $u \in \mathbf{C}_{0, T}^{2}$ and with probability one satisfies

$$
u_{t}(x)=u_{0}(x)+\int_{0}^{t}\left(\mathbf{L}_{s} u_{s}(x)+\mathbf{f}_{s}(x)\right) d s+\int_{0}^{t} \mathbf{G}_{\sigma_{s}^{k}} u_{s}(x) d W_{s}^{k}, \quad t \in[0, T], x \in \mathbb{R}^{d} .
$$

Such a solution is unique in the class of functions with quadratic exponential growth: precisely, $u$ is the unique solution such that there exists a positive random variable $C$ such that $\left|u_{t}(x)\right| e^{-C|x|^{2}}$ is bounded on $[\tau, T] \times \mathbb{R}^{d}$.

\subsection{Stochastic heat equation and Duhamel principle}

To further illustrate and motivate our results, in this section we consider the prototype case of the stochastic heat equation. We focus our attention on the Duhamel principle that is the crucial ingredient in the parametrix method for the construction of the fundamental solution. More generally, the Duhamel principle is a powerful tool for studying the existence and regularity properties of parabolic PDEs. In the framework of SPDEs of the form (1.2), it is still possible to have a Duhamel representation when the coefficients $\mathbf{a}^{i j}$ are deterministic and $\mathbf{G}_{\sigma_{t}^{k}} \equiv 0$ : this case has been considered in [26] and [18] where the Cauchy problem for parabolic SPDEs is studied. For the general SPDE (1.2) however, as 
also noticed by other authors (see, for instance, Sowers [27, Sect.3), measurability issues arise that do not appear in the deterministic case.

To be more specific, let us consider the stochastic heat equation

$$
d u_{t}(x)=\frac{\mathbf{a}^{2}}{2} \partial_{x x} u_{t}(x) d t+\left(\sigma \partial_{x} u_{t}(x)+\mathbf{g}_{t}(x)\right) d W_{t} .
$$

Under the coercivity condition $a^{2}:=\mathbf{a}^{2}-\sigma^{2}>0$, the Gaussian kernel

$$
p(t, x ; \tau, \xi):=\frac{1}{\sqrt{2 \pi a^{2}(t-\tau)}} \exp \left(-\frac{\left(x+\sigma\left(W_{t}-W_{\tau}\right)-\xi\right)^{2}}{2 a^{2}(t-\tau)}\right), \quad t>\tau \geq 0, x, \xi \in \mathbb{R},
$$

is well defined, and if $\sigma=0$ or $\mathbf{g} \equiv 0$ then the function

$$
u_{t}(x):=\int_{\mathbb{R}} p(t, x ; \tau, \xi) u_{0}(\xi) d \xi+\int_{0}^{t} \int_{\mathbb{R}} p(t, x ; s, \xi) \mathbf{g}_{s}(\xi) d \xi d W_{s}
$$

is a classical solution to (2.8), for any suitable initial value $u_{0}$. This follows directly from the Itô formula and the fact that the change of variable

$$
X_{\tau, t}(x)=x-\sigma\left(W_{t}-W_{\tau}\right)
$$

transforms (2.8) into a deterministic heat equation.

The difficulty in considering the case when $\sigma$ and $\mathbf{g}$ are both not null, comes from the fact that the integrand $p(t, x ; s, \xi) \mathbf{g}_{s}(\xi)$ in (2.10) becomes measurable with respect to the future $\sigma$-algebra $\mathcal{F}_{t}$ in the filtered space: thus in general the last integral in (2.10) is not well-defined in the framework of classical Itô-based stochastic calculus. For this reason, in the context of SPDEs, the Duhamel principle has been used only under rather specific assumptions.

We observe that a naive application of the parametrix method for SPDE (1.2) would consist precisely of a successive application of the Duhamel formula (2.10) with $\mathbf{g}$ and $\sigma=\sigma_{t}(x)$ that are not null and not even constant. Hence, the lack of a general Duhamel formula seems to preclude a direct use of the whole parametrix approach.

Incidentally formula (2.9) shows that, even for SPDEs with constant coefficients, the stochastic fundamental solution $p$ has distinctive properties compared to the Gaussian deterministic heat kernel. In particular, the asymptotic behaviour near the pole of $p$ is affected by the presence of the Brownian motion: this fact was studied also in [27] in the more general framework of Riemannian manifolds and is coherent with the Gaussian lower and upper bounds (2.5).

\section{Itô-Wentzell change of coordinates}

In this section we consider the random change of coordinates (2.2) and use the Itô-Wentzell formula to transform the SPDE (1.2) into a PDE with random coefficients. For simplicity, we only consider the case $\tau=0$ and set $X_{t}(x) \equiv X_{0, t}(x)$. We define the operation "hat" which transforms any function $u_{t}(x)$ into

$$
\hat{u}_{t}(x)=u_{t}\left(X_{t}(x)\right)
$$

and recall the classical Itô-Wentzell formula (see, for instance, Theor. 3.3 .1 in [17] or Theor. 6.4 in [14).

Theorem 3.1 (Itô-Wentzell). Let $u \in \mathbf{C}_{0, T}^{2}, h \in \mathbf{C}_{0, T}^{0}$ and $g^{k} \in \mathbf{C}_{0, T}^{1}$ be such that

$$
d u_{t}(x)=h_{t}(x) d t+g_{t}^{k}(x) d W_{t}^{k} .
$$


Then we have

$$
d \hat{u}_{t}(x)=\left(\hat{h}_{t}(x)+\frac{1}{2} \widehat{\sigma_{t}^{i k} \sigma_{t}^{j k}}(x) \widehat{\partial_{i j} u_{t}}(x)-\widehat{\partial_{i} g_{t}^{k}}(x) \hat{\sigma}_{t}^{i k}(x)\right) d t+\left(\hat{g}_{t}^{k}(x)-\widehat{\mathbf{G}_{\sigma_{t}^{k}} u_{t}}(x)\right) d W_{t}^{k} .
$$

In order to apply Itô-Wentzell formula to our SPDE, we prove the following crucial estimate for the gradient of $X_{t}(x)$.

Proposition 3.2. Let

$$
Y_{t}:=\left(\nabla X_{t}\right)^{-1}
$$

We have $\nabla X_{t}, Y_{t} \in \mathbf{b C}_{0, T}^{1}$ and there exists a positive random variable $\widetilde{\lambda}$ such that

$$
\left|Y_{t}^{*}(x) \xi\right|^{2} \geq \widetilde{\lambda}|\xi|^{2}, \quad t \in[0, T], x, \xi \in \mathbb{R}^{d}, P \text {-a.s. }
$$

The proof of Proposition 3.2 is based on the following preliminary lemma:

Lemma 3.3. Let $Z$ be a continuous random field defined on $[\tau, T] \times \mathbb{R}^{d}$. Assume that for some $\varepsilon>0$ and $p>\left(d \vee \frac{d}{2 \varepsilon}\right)$ there exists a constant $C>0$ such that

$$
\begin{gathered}
E\left[\sup _{s \in[\tau, T]}\left|Z_{s}(x)\right|^{p}\right] \leq C\left(1+|x|^{2}\right)^{-\varepsilon p}, \\
E\left[\sup _{s \in[\tau, T]}\left|\nabla Z_{s}(x)\right|^{p}\right] \leq C\left(1+|x|^{2}\right)^{-\varepsilon p},
\end{gathered}
$$

for every $x \in \mathbb{R}^{d}$. Then $Z$ has a modification in $\mathbf{b} \mathbf{C}_{\tau, T}^{1-\frac{d}{p}}$.

Proof. By the classical Sobolev embedding theorem, for every $f \in W^{1, p}\left(\mathbb{R}^{d}\right)$, with $p>d$, we have

$$
|f(x)|+\frac{|f(x)-f(y)|}{|x-y|^{1-\frac{d}{p}}} \leq N\|f\|_{W^{1, p}\left(\mathbb{R}^{d}\right)}, \quad \text { a.e. } x, y \in \mathbb{R}^{d},
$$

where $N$ is a constant dependent only on $p$ and $d$. Hence the statement directly follows from the following estimate

$$
\sup _{t \in[\tau, T]}\left\|Z_{t}\right\|_{W^{1, p}\left(\mathbb{R}^{d}\right)}<\infty \quad P \text {-a.e. }
$$

and the continuity of $Z$. To this end, we check that

$$
E\left[\sup _{t \in[\tau, T]}\left\|Z_{t}\right\|_{W^{1, p}\left(\mathbb{R}^{d}\right)}\right]<\infty
$$

By (3.5) and since $p>\frac{d}{2 \varepsilon}$, we have

$$
E\left[\sup _{t \in[\tau, T]}\left\|Z_{t}\right\|_{L^{p}\left(\mathbb{R}^{d}\right)}^{p}\right] \leq E\left[\int_{\mathbb{R}^{d}} \sup _{t \in[\tau, T]}\left|Z_{t}(x)\right|^{p} d x\right] \leq \int_{\mathbb{R}^{d}} C\left(1+|x|^{2}\right)^{-\varepsilon p} d x<\infty,
$$

and analogously by (3.6) we have

$$
E\left[\sup _{t \in[\tau, T]}\left\|\nabla Z_{t}\right\|_{L^{p}\left(\mathbb{R}^{d}\right)}^{p}\right] \leq \int_{\mathbb{R}^{d}} C\left(1+|x|^{2}\right)^{-\varepsilon p} d x<\infty .
$$


Proof of Proposition 3.2. Let

$$
Z_{t}(x):=\nabla X_{t}(x)-I=\int_{0}^{t} \nabla \sigma_{s}^{k}\left(X_{s}(x)\right) \nabla X_{s}(x) d W_{s}^{k} .
$$

We show that the matrix-valued random field $Z_{t}(x)$ satisfies estimates (3.5) and (3.6) of Lemma 3.3 for every $p$ such that $\left(2 \vee d \vee \frac{d}{2 \varepsilon}\right)<p<\bar{p}$, with $\varepsilon$ and $\bar{p}$ as in Assumption 2.4. Indeed, by the well-known $L^{p}$-estimates for $X_{t, T}(x)$ (see [17, Chapter 4), for any $0 \leq \tau \leq t \leq T$ and $x \in \mathbb{R}^{d}$ we have

$$
\begin{aligned}
& E\left[\left(1+\left|X_{\tau, t}(x)\right|^{2}\right)^{p}\right] \leq N_{1}\left(1+|x|^{2}\right)^{p}, \quad p \in \mathbb{R}, \\
& E\left[\left|\partial^{\beta} X_{\tau, t}(x)\right|^{p}\right] \leq N_{2}, \quad p \geq 2,1 \leq|\beta| \leq 3,
\end{aligned}
$$

where the constants $N_{1}$ and $N_{2}$ depend only on $p$ and $d$. We have

$$
E\left[\sup _{t \in[0, T]}\left|Z_{t}^{i j}(x)\right|^{p}\right] \leq C \sum_{k=1}^{d_{1}} \sum_{h=1}^{d} E\left[\sup _{t \in[0, T]}\left|\int_{0}^{t} \partial_{h} \sigma_{s}^{i k}\left(X_{s}(x)\right) \partial_{j} X_{s}^{h}(x) d W_{s}^{k}\right|^{p}\right]
$$

(by Burkolder inequality)

$$
\leq C_{p}^{\prime} \sum_{k=1}^{d_{1}} \sum_{h=1}^{d} E\left[\left(\int_{0}^{T}\left(\partial_{h} \sigma_{s}^{i k}\left(X_{s}(x)\right) \partial_{j} X_{s}^{h}(x)\right)^{2} d s\right)^{\frac{p}{2}}\right]
$$

(by Hölder inequality with conjugate exponents $\frac{p}{2}$ and $\frac{p}{p-2}$ )

$$
\leq C_{p}^{\prime} T^{\frac{p-2}{2}} \sum_{k=1}^{d_{1}} \sum_{h=1}^{d} \int_{0}^{T} E\left[\left|\partial_{h} \sigma_{s}^{i k}\left(X_{s}(x)\right) \partial_{j} X_{s}^{h}(x)\right|^{p}\right] d s
$$

(by Hölder inequality with conjugate exponents $r$ and $q<\frac{\bar{p}}{p}$ )

$$
\leq C_{p}^{\prime} T^{\frac{p-2}{2}} \sum_{k=1}^{d_{1}} \sum_{h=1}^{d} \int_{0}^{T} E\left[\left|\partial_{h} \sigma_{s}^{i k}\left(X_{s}(x)\right)\right|^{p q}\right]^{\frac{1}{q}} E\left[\left|\partial_{j} X_{s}^{h}(x)\right|^{p r}\right]^{\frac{1}{r}} d s
$$

(by Assumption 2.4 and estimate (3.9))

$$
\leq C_{p}^{\prime \prime} T^{\frac{p-2}{2}} N_{2}^{\frac{1}{r}} \int_{0}^{T} E\left[M^{p q}\left(1+\left|X_{s}(x)\right|^{2}\right)^{-\varepsilon p q}\right]^{\frac{1}{q}} d s
$$

(by Hölder inequality with conjugate exponents $\bar{r}$ and $\bar{q}:=\frac{\bar{p}}{p q}>1$ )

$$
\leq C_{p}^{\prime \prime} T^{\frac{p-2}{2}} N_{2}^{\frac{1}{r}} \int_{0}^{T} E\left[M^{\bar{p}}\right]^{\frac{p}{p}} E\left[\left(1+\left|X_{s}(x)\right|^{2}\right)^{-\varepsilon p q \bar{r}}\right]^{\frac{1}{q \bar{r}}} d s
$$

(by estimate (3.8))

$$
\leq C_{p}^{\prime \prime} T^{\frac{p}{2}} N_{1}^{\frac{1}{q \bar{r}}} N_{2}^{\frac{1}{r}}\|M\|_{\bar{p}}^{p}\left(1+|x|^{2}\right)^{-\varepsilon p} .
$$

This proves (3.5). Estimate (3.6) is obtained in a similar way from the identity $\partial_{h} Z_{t}^{i j}(x)=\partial_{h j}^{2} X_{t}^{i}$, with $\partial_{h j}^{2} X_{t}^{i}$ satisfying SDE (2.4), and employing estimate (3.9) with $|\beta|=2$. Hence, by Lemma 3.3, $Z_{t}(x)$ has a $\mathbf{b C}_{0, T}^{1-\frac{d}{p}}$-modification and therefore $\nabla X_{t}(x)$ is bounded as a function of $(t, x) \in[0, T] \times \mathbb{R}^{d}$, $P$-a.e. by (3.7).

Next we prove that $\operatorname{det} \nabla X_{t}(x)$ is bounded from above and below by a positive random variable for all $(t, x), P$-a.s. By Itô formula (see [14, Lemma 3.1 for more details), with probability one we have

$$
\operatorname{det} \nabla X_{t}(x)=\exp \left(-\int_{0}^{t} \operatorname{tr} D \sigma_{s}^{k}\left(X_{s}(x)\right) d W_{s}^{k}+\frac{1}{2} \int_{0}^{t} \operatorname{tr}\left(\left(D \sigma_{s}^{k}\right)^{2}\right)\left(X_{s}(x)\right) d s\right) .
$$


Since both parts of the equality are continuous w.r.t $(t, x)$, the equality holds for all $(t, x)$ at once with probability one. Thus the assertion follows from the boundedness of the integrals appearing in (3.10), which again can be proved as an application of Lemma 3.3. estimate (3.8) and Assumption 2.4.

Then the matrix $Y_{t}(x)$ is well defined and $\operatorname{det} Y_{t}(x)$ is bounded from below by a positive random variable for all $(t, x), P$-a.s. This fact, together with the uniform boundedness of the entries of $\nabla X_{t}(x)$, implies (3.4).

It remains to prove that $\nabla X_{t}$ and $Y_{t}$ have uniformly bounded spatial derivatives $P$-a.s. Again, this is a consequence of formula (2.4), Lemma 3.3 and the simple equality $\partial_{j} Y_{t}(x)=-Y_{t}(x) \partial_{j}\left(\nabla X_{t}(x)\right) Y_{t}(x)$.

Theorem 3.4. The function $u$ is a classical solution of SPDE (1.2) if and only if $\hat{u}$ in (3.1) solves

$$
d \hat{u}_{t}(x)=\left(L_{t} \hat{u}_{t}(x)+f_{t}(x)\right) d t
$$

where $f_{t}=\hat{\mathbf{f}}_{t}$ and

$$
L_{t}=\frac{1}{2} a_{t}^{i j} \partial_{i j}+b_{t}^{i} \partial_{i}+c_{t}
$$

is the parabolic operator with coefficients $a^{i j}, b^{j}, c \in \mathbf{b C}_{0, T}^{\alpha}$ given explicitly by

$$
a_{t}^{i j}=\left(Y_{t} \hat{\mathbf{A}}_{t} Y_{t}^{*}\right)_{i j}, \quad b_{t}^{i}=Y_{t}^{i r}\left(\hat{\mathbf{b}}_{t}^{r}-\partial_{j} \widehat{\sigma_{t}^{r k} \sigma_{t}^{j k}}-\hat{\mathbf{a}}_{t}^{j h}\left(Y_{t}^{*}\left(\nabla^{2} X_{t}^{r}\right) Y_{t}\right)_{j h}\right), \quad c_{t}=\hat{\mathbf{c}}_{t} .
$$

Moreover, for some positive random variable $\mu$, the following coercivity condition is satisfied

$$
\left\langle a_{t}(x) \xi, \xi\right\rangle \geq \mu|\xi|^{2}, \quad t \in[0, T], x, \xi \in \mathbb{R}^{d}, P \text {-a.s. }
$$

Proof. By assumption, $u_{t}$ satisfies (3.2) with $h_{t}=\mathbf{L}_{t} u_{t}+\mathbf{f}_{t} \in \mathbf{C}_{0, T}^{\alpha}$ and $g_{t}^{k}=\mathbf{G}_{\sigma_{t}^{k}} u_{t} \in \mathbf{C}_{0, T}^{1+\alpha}$. Thus, by the Itô-Wentzell formula (3.3) we get

$$
d \hat{u}_{t}=\left(\frac{1}{2} \hat{\mathbf{A}}_{t}^{i j} \widehat{\partial_{i j} u_{t}}+\left(\hat{\mathbf{b}}_{t}^{j}-\widehat{\partial_{i} \sigma_{t}^{j k} \sigma_{t}^{i k}}\right) \widehat{\partial}_{j} u_{t}+\hat{\mathbf{c}}_{t} \hat{u}_{t}+\hat{\mathbf{f}}\right) d t .
$$

Now, we have

$$
\begin{aligned}
\partial_{j} \hat{u}_{t}(x) & =\widehat{\partial}_{i} u_{t}(x) \partial_{j} X_{t}^{i}(x)=\left(\widehat{\nabla u_{t}}(x) \nabla X_{t}(x)\right)_{j}, \\
\partial_{i j} \hat{u}_{t}(x) & =\left(\nabla X_{t}^{*}(x) \widehat{\nabla^{2} u_{t}}(x) \nabla X_{t}(x)\right)_{i j}+\left(\widehat{\partial_{h} u_{t}}(x) \nabla^{2} X_{t}^{h}(x)\right)_{i j},
\end{aligned}
$$

or equivalently

$$
\begin{aligned}
\widehat{\nabla u_{t}}(x) & =\nabla \hat{u}_{t}(x) Y_{t}(x), \\
\widehat{\nabla^{2} u_{t}}(x) & =Y_{t}^{*}(x) \nabla^{2} \hat{u}_{t}(x) Y_{t}(x)-\left(Y_{t}^{*}(x) \nabla^{2} X_{t}^{h}(x) Y_{t}(x)\right) \widehat{\partial_{h} u_{t}}(x) .
\end{aligned}
$$

Plugging these formulas into (3.15) and rearranging the indexes, we get (3.11)-(3.12)-(3.13). Moreover, from expressions (3.13) combined with Assumption 2.3 and Proposition 3.2 it is straightforward to see that $a^{i j}, b^{j}, c \in \mathbf{b C}_{0, T}^{\alpha}$. Eventually, by Assumption 2.2 and estimate (3.4) of Proposition 3.2 we have

$$
\left\langle\hat{\mathbf{A}}_{t}(x) Y_{t}^{*} \xi, Y_{t}^{*} \xi\right\rangle \geq \lambda\left|Y_{t}^{*}(x) \xi\right|^{2} \geq \lambda \widetilde{\lambda}|\xi|^{2}
$$

for any $t \in[0, T], x, \xi \in \mathbb{R}^{d}, P$-a.s. and this proves (3.14). 


\section{Time-dependent parametrix}

In this section we consider the (deterministic) parabolic PDE

$$
\mathcal{H} u_{t}(x):=L_{t} u_{t}(x)-\partial_{t} u_{t}(x)=0
$$

where

$$
L_{t} u_{t}(x)=\frac{1}{2} a_{t}^{i j} \partial_{i j} u_{t}(x)+b_{t}^{i} \partial_{i} u_{t}(x)+c_{t} u_{t}(x)
$$

appears in the reduced equation (3.11) when $\omega \in \Omega$ is fixed. Since the coefficients will be assumed only measurable in the time variable, equation (4.1) has to be understood in the integral sense: a solution to the Cauchy problem

$$
\begin{cases}\mathcal{H} u_{t}(x)+f_{t}(x)=0, & x \in \mathbb{R}^{d}, \text { a.e. } t \in(\tau, T], \\ u_{\tau}(x)=\varphi(x), & x \in \mathbb{R}^{d},\end{cases}
$$

is a function $u \in C_{\tau, T}^{2}\left(\mathbb{R}^{d}\right)$ that satisfies

$$
u_{t}(x)=\varphi(x)+\int_{\tau}^{t}\left(L_{s} u_{s}(x)+f_{s}(x)\right) d s, \quad(t, x) \in[\tau, T] \times \mathbb{R}^{d} .
$$

The main idea of the parametrix method is to construct the fundamental solution $\Gamma=\Gamma(t, x ; \tau, \xi)$ of $\mathcal{H}$ using as a first approximation the so-called parametrix, that is the Gaussian kernel of the heat operator obtained by freezing the coefficients of $\mathcal{H}$ at the pole $(\tau, \xi)$. If $Z=Z(t, x ; \tau, \xi)$ denotes the parametrix, one looks for the fundamental solution of $\mathcal{H}$ in the form

$$
\Gamma(t, x ; \tau, \xi)=Z(t, x ; \tau, \xi)+\int_{\tau}^{t} \int_{\mathbb{R}^{d}} Z(t, x ; s, y) \Phi(s, y ; \tau, \xi) d y d s .
$$

The unknown function $\Phi$ is determined by imposing $\mathcal{H} \Gamma(t, x ; \tau, \xi)=0$ : this implies that $\Phi$ should satisfy the integral equation

$$
\Phi(t, x ; \tau, \xi)=\mathcal{H} Z(t, x ; \tau, \xi)+\int_{\tau}^{t} \int_{\mathbb{R}^{d}} \mathcal{H} Z(t, x ; s, y) \Phi(s, y ; \tau, \xi) d y d s
$$

for any $x, \xi \in \mathbb{R}^{d}$ and a.e. $t \in(\tau, T]$. By recursive approximation we have

$$
\Phi(t, x ; \tau, \xi)=\sum_{k=1}^{+\infty}(\mathcal{H} Z)_{k}(t, x ; \tau, \xi)
$$

where

$$
\begin{aligned}
(\mathcal{H} Z)_{1}(t, x ; \tau, \xi) & =\mathcal{H} Z(t, x ; \tau, \xi), \\
(\mathcal{H} Z)_{k+1}(t, x ; \tau, \xi) & =\int_{\tau}^{t} \int_{\mathbb{R}^{d}} \mathcal{H} Z(t, x ; s, y)(\mathcal{H} Z)_{k}(s, y ; \tau, \xi) d y d s, \quad k \in \mathbb{N} .
\end{aligned}
$$

To prove convergence of the series (4.6) and show that the candidate $\Gamma$ in (4.4)-(4.5) is indeed a fundamental solution for $\mathcal{H}$, we need to impose some conditions.

Assumption 4.1 (Coercivity). There exists a positive constant $\lambda$ such that

$$
\lambda^{-1}|\xi|^{2} \leq\left\langle a_{t}(x) \xi, \xi\right\rangle \leq \lambda|\xi|^{2}, \quad t \in[0, T], x, \xi \in \mathbb{R}^{d} .
$$

Assumption 4.2 (Regularity). The coefficients $a^{i j}, b^{j}, c$ are bounded functions and $a^{i j} \in C_{0, T}^{\alpha}\left(\mathbb{R}^{d}\right)$, $b^{j}, c \in C_{0, T, \text { loc }}^{\alpha}\left(\mathbb{R}^{d}\right)$ for some $\alpha \in(0,1)$. 
Since it is clearly not restrictive, for the subsequent analysis it is convenient to increase a bit the value of the constant $\lambda$ of Assumption 4.1 so that $\lambda>1$ and the $L^{\infty}$-norms of the coefficients $a^{i j}, b^{j}, c$ are bounded by $\lambda$.

Remark 4.3. As opposed to the classical parametrix method, in Assumption 4.2 we do not require any regularity of the coefficients in the time variable. Instead, here we only require Hölder continuity in the spatial variables. The reason lies in the fact that we are going to adopt a time-dependent definition of parametrix: namely, we do not freeze the time variable in the definition of $Z$ (see (4.13) below) and take as parametrix the fundamental solution of a parabolic equation with coefficients depending on $t$.

Remark 4.4. Using the enhanced version of the parametrix method proposed in [2], we can weaken the conditions on the first- and zero-order coefficients that can be supposed to be unbounded with sub-linear growth at infinity.

Definition 4.5. A fundamental solution $\Gamma=\Gamma(t, x ; \tau, \xi)$ for equation (4.1) is a function defined for $0 \leq \tau<t \leq T$ and $x, \xi \in \mathbb{R}^{d}$, such that for any $(\tau, \xi) \in[0, T) \times \mathbb{R}^{d}$ we have:

i) $\Gamma(\cdot, \cdot ; \tau, \xi) \in C_{t_{0}, T}^{2}\left(\mathbb{R}^{d}\right)$ for any $\left.t_{0} \in\right] \tau, T\left[\right.$ and satisfies $\mathcal{H} \Gamma(t, x ; \tau, \xi)=0$ for any $x \in \mathbb{R}^{d}$ and a.e. $t \in(\tau, T]$

ii) for any continuous and non-rapidly increasing function $\varphi$ on $\mathbb{R}^{d}$

$$
\lim _{\substack{(t, x \rightarrow(\tau, \xi) \\ t>\tau}} \int_{\mathbb{R}^{d}} \Gamma(t, x ; \tau, y) \varphi(y) d y=\varphi(\xi) .
$$

Next we state the main result of this section.

Theorem 4.6 (Existence of the fundamental solution). Under Assumptions 4.1] and 4.2, there exists a fundamental solution $\Gamma$ for equation (4.1). Moreover, assume that $\varphi=\varphi(x)$ is continuous and non-rapidly increasing on $\mathbb{R}^{d}$, and $f=f_{t}(x)$ is non-rapidly increasing uniformly on $[\tau, T] \times \mathbb{R}^{d}$ and such that $f \in C_{\tau, T, \text { loc }}^{\alpha^{\prime}}$ for some $\alpha^{\prime} \in(0,1)$. Then

$$
u_{t}(x)=\int_{\mathbb{R}^{d}} \Gamma(t, x ; \tau, \xi) \varphi(\xi) d \xi+\int_{\tau}^{t} \int_{\mathbb{R}^{d}} \Gamma(t, x ; s, \xi) f(s, \xi) d \xi d s
$$

is a solution to the Cauchy problem (4.3). Such a solution is unique in the class of functions with quadratic exponential growth (cf. Corollary [2.6).

Theorem 4.7 (Properties of the fundamental solution). Under the same assumptions of Theorem 4.6. the fundamental solution $\Gamma$ enjoys the following properties:

i) $\Gamma$ verifies the Chapman-Kolmogorov identity

$$
\Gamma\left(t, x ; t_{0}, x_{0}\right)=\int_{\mathbb{R}^{d}} \Gamma(t, x ; \tau, \xi) \Gamma\left(\tau, \xi ; t_{0}, x_{0}\right) d \xi, \quad t_{0}<\tau<t, x, x_{0} \in \mathbb{R}^{d} ;
$$

and, if $c=c_{t}$ is independent of $x$, we have

$$
\int_{\mathbb{R}^{d}} \Gamma(t, x ; \tau, \xi) d \xi=e^{\int_{\tau}^{t} c_{s} d s}, \quad \tau \leq t \leq T, x \in \mathbb{R}^{d} .
$$

In particular, if $c \equiv 0$ then $\Gamma(t, x ; \tau, \cdot)$ is a density; 
ii) there exist two positive constants $C_{i}=C_{i}(\lambda, \alpha, d, T), i=1,2$, such that

$$
\begin{aligned}
\frac{1}{C_{1}} \Gamma^{C_{2}}(t-\tau, x-\xi) & \leq \Gamma(t, x ; \tau, \xi) \leq C_{1} \Gamma^{\lambda}(t-\tau, x-\xi), \\
\left|\partial_{x_{i}} \Gamma(t, x ; \tau, \xi)\right| & \leq \frac{C_{1}}{\sqrt{t-\tau}} \Gamma^{\lambda}(t-\tau, x-\xi), \\
\left|\partial_{x_{i} x_{j}} \Gamma(t, x ; \tau, \xi)\right| & \leq \frac{C_{1}}{t-\tau} \Gamma^{\lambda}(t-\tau, x-\xi),
\end{aligned}
$$

for every $0 \leq \tau<t \leq T$ and $x, \xi \in \mathbb{R}^{d}$, where $\Gamma^{\lambda}$ denotes the fundamental solution of the heat equation $\partial_{t} u_{t}(x)=\frac{\lambda}{2} \Delta u_{t}(x)$.

\subsection{Preliminary Gaussian and potential estimates}

Let $A=\left(A^{i j}\right)_{1 \leq i, j \leq d}$ be a constant, symmetric and positive definite matrix. We denote by

$$
\Gamma^{\text {heat }}(A, x)=\frac{1}{\sqrt{(2 \pi)^{d} \operatorname{det} A}} e^{-\frac{1}{2}\left\langle A^{-1} x, x\right\rangle}, \quad x \in \mathbb{R}^{d},
$$

the $d$-dimensional Gaussian kernel with covariance matrix $A$. Clearly $\Gamma^{\text {heat }}$ is a smooth function and satisfies

$$
\partial_{t} \Gamma^{\text {heat }}(t A, x)=\frac{1}{2} \sum_{i, j=1}^{d} A^{i j} \partial_{i j} \Gamma^{\text {heat }}(t A, x), \quad t>0, x \in \mathbb{R}^{d} .
$$

Now, we freeze the coefficients of $L_{t}$ in (4.2) at a fixed point $y \in \mathbb{R}^{d}$ and consider the operator with time-dependent coefficients

$$
L_{t, y}=\frac{1}{2} a_{t}^{i j}(y) \partial_{x_{i} x_{j}}
$$

acting in the $x$-variable. We denote by

$$
\Gamma_{y}(t, x ; \tau, \xi)=\Gamma^{\text {heat }}\left(A_{\tau, t}(y), x-\xi\right), \quad A_{\tau, t}(y):=\int_{\tau}^{t} a_{s}(y) d s,
$$

the fundamental solution of $L_{t, y}-\partial_{t}$. Notice that $\Gamma_{y}$ is well defined for $0 \leq \tau<t \leq T$ in virtue of Assumption 4.1 and solves

$$
\partial_{t} \Gamma_{y}(t, x ; \tau, \xi)=L_{t, y} \Gamma_{y}(t, x ; \tau, \xi)
$$

for any $x, \xi \in \mathbb{R}^{d}$ and almost every $t \in(\tau, T]$. Finally, we define the parametrix for $\mathcal{H}$ as

$$
Z(t, x ; \tau, \xi)=\Gamma_{\xi}(t, x ; \tau, \xi), \quad 0 \leq \tau<t \leq T, x, \xi \in \mathbb{R}^{d} .
$$

Hereafter $C=C(\cdot, \ldots, \cdot)$ denotes a constant depending only on quantities appearing in parentheses. In a given context the same letter will be used to denote different constants depending on the same set of arguments. The following Gaussian estimates are standard consequences of Assumption 4.1

Lemma 4.8. We have

$$
\frac{1}{\lambda^{d}} \Gamma^{\frac{1}{\lambda}}(t-\tau, x-\xi) \leq \Gamma_{y}(t, x ; \tau, \xi) \leq \lambda^{d} \Gamma^{\lambda}(t-\tau, x-\xi),
$$

for any $0 \leq \tau<t \leq T$ and $x, \xi, y \in \mathbb{R}^{d}$. Moreover, $\Gamma_{y}(t, x ; \tau, \xi)$ verifies the Gaussian estimates (4.10)-(4.11) for some positive constant $C=C(\lambda, d)$. 
Proposition 4.9. There exists $k_{0} \in \mathbb{N}$ such that, for every $\tau \in\left[0, T\left[\right.\right.$ and $\xi \in \mathbb{R}^{d}$, the series

$$
\sum_{k=k_{0}}^{\infty}(\mathcal{H} Z)_{k}(\cdot, \cdot ; \tau, \xi)
$$

converges in $L^{\infty}\left((\tau, T] \times \mathbb{R}^{d}\right)$. The function $\Phi$ defined by (4.6) solves the integral equation (4.5) and there exists a positive constant $C=C(\lambda, \alpha, d, T)$ such that

$$
\begin{aligned}
|\Phi(t, x ; \tau, \xi)| & \leq \frac{C}{(t-\tau)^{1-\frac{\alpha}{2}}} \Gamma^{\lambda}(t-\tau, x-\xi), \\
|\Phi(t, x ; \tau, \xi)-\Phi(t, y ; \tau, \xi)| & \leq C \frac{|x-y|^{\frac{\alpha}{2}}}{(t-\tau)^{1-\frac{\alpha}{4}}}\left(\Gamma^{\lambda}(t-\tau, x-\xi)+\Gamma^{\lambda}(t-\tau, y-\xi)\right),
\end{aligned}
$$

for every $x, y, \xi \in \mathbb{R}^{d}$ and almost every $t \in(\tau, T]$.

Proof. We prove the preliminary estimate

$$
\left|(\mathcal{H} Z)_{k}(t, x ; \tau, \xi)\right| \leq \frac{M_{k}}{(t-\tau)^{1-\alpha k / 2}} \Gamma^{\lambda}(t-\tau, x-\xi) \quad x, \xi \in \mathbb{R}^{d}, \text { a.e. } t \in(\tau, T], k \in \mathbb{N},
$$

where $C=C(\lambda, \alpha, d, T)$ is a positive constant, $M_{k}=C^{k} \frac{\Gamma_{E}^{k}\left(\frac{\alpha}{2}\right)}{\Gamma_{E}\left(\frac{\alpha k}{2}\right)}$ and $\Gamma_{E}$ is the Euler Gamma function.

For $k=1$, we have

$$
|\mathcal{H} Z(t, x ; \tau, \xi)|=\left|\left(L_{t}-L_{t, \xi}\right) Z(t, x ; \tau, \xi)\right| \leq I_{1}+I_{2}+I_{3}
$$

where

$$
I_{1}=\frac{1}{2}\left|a_{t}^{i j}(x)-a_{t}^{i j}(\xi)\right|\left|\partial_{i j} Z(t, x ; \tau, \xi)\right|, \quad I_{2}=\left|b_{t}^{i}(x)\right|\left|\partial_{i} Z(t, x ; \tau, \xi)\right|, \quad I_{3}=\left|c_{t}(x) Z(t, x ; \tau, \xi)\right| .
$$

By Assumption 4.2 and Lemma 4.8, we have

$$
I_{1} \leq \frac{C}{(t-\tau)^{1-\frac{\alpha}{2}}}\left(\frac{|x-\xi|}{\sqrt{t-\tau}}\right)^{\alpha} \Gamma^{\lambda}(t-\tau, x-\xi) \leq \frac{C}{(t-\tau)^{1-\frac{\alpha}{2}}} \Gamma^{\lambda+1}(t-\tau, x-\xi) .
$$

Since the coefficients are bounded, by Lemma 4.8 we also have

$$
I_{2} \leq \frac{C}{\sqrt{t-\tau}} \Gamma^{\lambda}(t-\tau, x-\xi) \leq C \frac{\Gamma^{\lambda}(t-\tau, x-\xi)}{(t-\tau)^{1-\frac{\alpha}{2}}}, \quad I_{3} \leq C \Gamma^{\lambda}(t-\tau, x-\xi),
$$

and this proves (4.17) for $k=1$. Now we assume that (4.17) holds for $k$ and prove it for $k+1$ : we have

$$
\left|(\mathcal{H} Z)_{k+1}(t, x ; \tau, \xi)\right|=\left|\int_{\tau}^{t} \int_{\mathbb{R}^{d}} \mathcal{H} Z(t, x ; s, y)(\mathcal{H} Z)_{k}(s, y ; \tau, \xi) d y d s\right| \leq
$$

(by inductive hypothesis)

$$
\leq \int_{\tau}^{t} \frac{M_{1}}{(t-s)^{1-\alpha / 2}} \frac{M_{k}}{(s-\tau)^{1-\alpha k / 2}} \int_{\mathbb{R}^{d}} \Gamma^{\lambda}(t-s, x-y) \Gamma^{\lambda}(s-\tau, y-\xi) d y d s \leq
$$

(by the Chapman-Kolmogorov property for $\Gamma^{\lambda}$ )

$$
\leq \Gamma^{\lambda}(t-\tau, x-\xi) \int_{\tau}^{t} \frac{M_{1}}{(t-s)^{1-\alpha / 2}} \frac{M_{k}}{(s-\tau)^{1-\alpha k / 2}} d s
$$

that yields (4.17) thanks to the well-known properties of the Gamma function. From (4.17) we directly deduce the uniform convergence of the series and estimate (4.15). The proof of (4.16) follows the same lines as in the classical case (see [7, Ch.1, Theor.7) and is omitted. 
We close this section by stating a generalization of a classical result about the so-called volume potential defined as

$$
V_{f}(t, x)=\int_{t_{0}}^{t} \int_{\mathbb{R}^{d}} Z(t, x ; \tau, \xi) f(\tau, \xi) d \xi d \tau, \quad(t, x) \in\left[t_{0}, T\right] \times \mathbb{R}^{d},
$$

where $Z$ denotes the parametrix. The proof is based on classical arguments (see [7, Ch.1, Sec.3 and 9]) that can be applied to the time-dependent parametrix $Z$ in (4.13) without any significant change.

Lemma 4.10. Let $V_{f}$ be the volume potential in (4.18) with $f \in C_{t_{0}, T, \text { loc }}^{\alpha}\left(\mathbb{R}^{d}\right)$, non-rapidly increasing uniformly w.r.t. t. Then $V_{f} \in C_{t_{0}, T}^{2}\left(\mathbb{R}^{d}\right)$ satisfies

$$
\begin{aligned}
\partial_{x_{i}} V_{f}(t, x) & =\int_{t_{0}}^{t} \int_{\mathbb{R}^{d}} \partial_{x_{i}} Z(t, x ; \tau, \xi) f(\tau, \xi) d \xi d \tau, \\
\partial_{x_{i} x_{j}} V_{f}(t, x) & =\int_{t_{0}}^{t} \int_{\mathbb{R}^{d}} \partial_{x_{i} x_{j}} Z(t, x ; \tau, \xi) f(\tau, \xi) d \xi d \tau, \\
\partial_{t} V_{f}(t, x) & =f(t, x)+\int_{t_{0}}^{t} \int_{\mathbb{R}^{d}} \partial_{t} Z(t, x ; \tau, \xi) f(\tau, \xi) d \xi d \tau,
\end{aligned}
$$

for any $x \in \mathbb{R}^{d}$ and a.e. $t \in\left(t_{0}, T\right]$.

\subsection{Proof of Theorem 4.6}

Let $\Gamma=\Gamma(t, x ; \tau, \xi)$ be the function defined by (4.4)-(4.6) for $0 \leq \tau<t \leq T$ and $x, \xi \in \mathbb{R}^{d}$. By Proposition 4.9], it is clear that $\Gamma(\cdot, \cdot ; \tau, \xi) \in C_{\tau, T}^{0}\left(\mathbb{R}^{d}\right)$ for any $(\tau, \xi) \in[0, T) \times \mathbb{R}^{d}$. Next, we fix $t_{0} \in(\tau, t)$ and notice that by (4.15)-(4.16) the function $f:=\Phi(\cdot, \cdot ; \tau, \xi)$, defined on $\left[t_{0}, T\right] \times \mathbb{R}^{d}$, satisfies the conditions of Lemma 4.10 hence the volume potential

$$
V_{\Phi}(t, x):=\int_{t_{0}}^{t} \int_{\mathbb{R}^{d}} Z(t, x ; s, y) \Phi(s, y ; \tau, \xi) d y d s
$$

is twice continuously differentiable in $x$ and satisfies

$$
\mathcal{H} V_{\Phi}(t, x)=\int_{t_{0}}^{t} \int_{\mathbb{R}^{d}} \mathcal{H} Z(t, x ; s, y) \Phi(s, y ; \tau, \xi) d y d s-\Phi(t, x ; \tau, \xi), \quad \text { a.e. } t \in\left(t_{0}, T\right] .
$$

On the other hand, we also have

$$
\mathcal{H} \int_{\tau}^{t_{0}} \int_{\mathbb{R}^{d}} Z(t, x ; s, y) \Phi(s, y ; \tau, \xi) d y d s=\int_{\tau}^{t_{0}} \int_{\mathbb{R}^{d}} \mathcal{H} Z(t, x ; s, y) \Phi(s, y ; \tau, \xi) d y d s
$$

by the dominated convergence theorem. Consequently, we have

$$
\mathcal{H} \Gamma(t, x ; \tau, \xi)=\mathcal{H} Z(t, x ; \tau, \xi)+\int_{\tau}^{t} \int_{\mathbb{R}^{d}} \mathcal{H} Z(t, x ; s, y) \Phi(s, y ; \tau, \xi) d y d s-\Phi(t, x ; \tau, \xi)=0
$$

for a.e. $t \in(\tau, T]$, because $\Phi$ solves equation (4.5). This proves property i) of Definition 4.5 of fundamental solution. To prove property ii), it suffices to notice that

$$
\int_{\mathbb{R}^{d}} \Gamma(t, x ; \tau, y) \varphi(y) d y=I_{1}(t, x, \tau)+I_{2}(t, x, \tau)
$$

where

$$
\lim _{\substack{(t, x) \rightarrow(\tau, \xi) \\ t>\tau}} I_{1}(t, x, \tau)=\lim _{\substack{(t, x) \rightarrow(\tau, \xi) \\ t>\tau}} \int_{\mathbb{R}^{d}} Z(t, x ; \tau, y) \varphi(y) d y=\varphi(\xi),
$$




$$
\lim _{\substack{(t, x) \rightarrow(\tau, \xi) \\ t>\tau}}\left|I_{2}(t, x, \tau)\right| \leq \lim _{\substack{(t, x) \rightarrow(\tau, \xi) \\ t>\tau}} \int_{\mathbb{R}^{d}} \int_{\tau}^{t} \int_{\mathbb{R}^{d}} Z(t, x ; s, \eta)|\Phi(s, \eta ; \tau, y) \varphi(y)| d \eta d s d y \leq
$$

(by (4.14)-(4.15) and since $\varphi$ is non-rapidly increasing, taking $\delta>0$ suitably small, with $C=C(\lambda, \delta)$ )

$$
\begin{aligned}
& \leq \lim _{\substack{(t, x) \rightarrow(\tau, \xi) \\
t>\tau}} \int_{\mathbb{R}^{d}} \int_{\tau}^{t} \frac{C}{(s-\tau)^{1-\frac{\alpha}{2}}} \int_{\mathbb{R}^{d}} \Gamma^{\lambda}(t-s, x-\eta) \Gamma^{\lambda}(s-\tau, \eta-y) e^{\delta|y|^{2}} d \eta d s d y \\
& \leq \lim _{\substack{(t, x) \rightarrow(\tau, \xi) \\
t>\tau}} \int_{\mathbb{R}^{d}} \int_{\tau}^{t} \frac{C}{(s-\tau)^{1-\frac{\alpha}{2}}} \Gamma^{\lambda}(t-\tau, x-y) e^{\delta|y|^{2}} d s d y=0 .
\end{aligned}
$$

Finally, the standard proof of existence for the Cauchy problem (see for instance [7, Ch.1, Theor.12, or [9]) applies without modification. Uniqueness follows from the maximum principle.

\subsection{Proof of Theorem 4.7}

The Chapman-Kolmogorov identity follows from uniqueness of the Cauchy problem (4.3) and representation (4.7) with $f \equiv 0$ and $\varphi=\Gamma\left(\tau, \cdot ; \tau_{0}, \xi_{0}\right)$, for fixed $\left(\tau_{0}, \xi_{0}\right) \in[0, \tau) \times \mathbb{R}^{d}$. Analogously, formula (4.8) follows from uniqueness of the Cauchy problem (4.3) with $f \equiv 0$ and $\varphi \equiv 1$.

Next we prove the Gaussian estimates for $\Gamma$. First, we set

$$
J(t, x ; \tau, \xi)=\int_{\tau}^{t} \int_{\mathbb{R}^{d}} Z(t, x ; s, y) \Phi(s, y ; \tau, \xi) d y d s
$$

and notice that

$$
|J(t, x ; \tau, \xi)| \leq \int_{\tau}^{t} \int_{\mathbb{R}^{d}} Z(t, x ; s, y)|\Phi(s, y ; \tau, \xi)| d y d s \leq
$$

(by (4.14), (4.15) and the Chapman-Kolmogorov property for $\Gamma^{\lambda}$ )

$$
\leq \Gamma^{\lambda}(t-\tau, x-\xi) \int_{\tau}^{t} \frac{C}{(s-\tau)^{1-\frac{\alpha}{2}}} d s \leq C(t-\tau)^{\frac{\alpha}{2}} \Gamma^{\lambda}(t-\tau, x-\xi)
$$

for some positive $C=C(\lambda, d, T)$. Since $\Gamma=Z+J$, the previous estimate combined with (4.14) proves

$$
|\Gamma(t, x ; \tau, \xi)| \leq C_{1} \Gamma^{\lambda}(t-\tau, x-\xi)
$$

and in particular, the upper bound for $\Gamma$ in (4.9). The proof of (4.10)-(4.11) is similar. Notice that by the maximum principle (in the form of Lemma 5 p.43 in [7]) applied to $u(t, x)=\int_{\mathbb{R}^{d}} \Gamma(t, x ; \tau, y) \varphi(y) d y$, where $\varphi$ is any bounded, non-negative and continuous function, one easily infers that $\Gamma$ is non-negative.

To prove the Gaussian lower bound we adapt a procedure due to Aronson that is essentially based on a crucial Nash's lower bound (see [5, Sect. 2). The main difference is that in our setting we replace Nash's estimate with a bound that we directly derive from the parametrix method. Let us first notice that, for $\lambda>1$, we have $\Gamma^{\lambda}(t ; x) \leq \Gamma^{\frac{1}{\lambda}}(t ; x)$ if $|x| \leq \varrho_{\lambda} \sqrt{t}$ where $\varrho_{\lambda}=\sqrt{\frac{\lambda d}{\lambda^{2}-1} \log \lambda}$. Thus, by (4.14) and (4.20) we have

$$
\Gamma(t, x ; \tau, \xi) \geq Z(t, x ; \tau, \xi)-|J(t, x ; \tau, \xi)| \geq
$$

(if $|x-\xi| \leq \varrho_{\lambda} \sqrt{t-\tau}$ )

$$
\begin{aligned}
& \geq\left(\lambda^{-d}-C(t-\tau)^{\frac{\alpha}{2}}\right) \Gamma^{\frac{1}{\lambda}}(t-\tau ; x-\xi) \\
& \geq \frac{1}{2 \lambda^{d}} \Gamma^{\frac{1}{\lambda}}(t-\tau ; x-\xi)
\end{aligned}
$$


if $0<t-\tau \leq T_{\lambda}:=\left(2 C \lambda^{d}\right)^{-\frac{2}{\alpha}} \wedge T$.

For any $(t, x),(\tau, \xi) \in[0, T] \times \mathbb{R}^{d}$, we set $m$ to be the smallest natural number greater than

$$
\max \left\{4 \varrho_{\lambda}^{-2} \frac{|x-\xi|^{2}}{(t-\tau)}, \frac{T}{T_{\lambda}}\right\}
$$

Then we set

$$
t_{i}=\tau+i \frac{t-\tau}{m+1}, \quad x_{i}=\xi+i \frac{x-\xi}{m+1}, \quad i=0, \ldots, m+1 .
$$

Denoting by $D(x ; r)=\left\{y \in \mathbb{R}^{d}|| x-y \mid<r\right\}$ the Euclidean ball centered at $x$ with radius $r>0$, by the Chapman-Kolmogorov equation we have

$\Gamma(t, x ; \tau, \xi)=\int_{\mathbb{R}^{m d}} \Gamma\left(t, x ; t_{m}, y_{m}\right) \prod_{i=1}^{m-1} \Gamma\left(t_{i+1}, y_{i+1} ; t_{i}, y_{i}\right) \Gamma\left(t_{1}, y_{1} ; \tau, \xi\right) d y_{1} \cdots d y_{m}$

(since $\Gamma$ is non-negative)

$$
\geq \int_{\mathbb{R}^{m d}} \Gamma\left(t, x ; t_{m}, y_{m}\right) \mathbb{1}_{D\left(x_{m} ; r\right)}\left(y_{m}\right) \prod_{i=1}^{m-1} \Gamma\left(t_{i+1}, y_{i+1} ; t_{i}, y_{i}\right) \mathbb{1}_{D\left(x_{i} ; r\right)}\left(y_{i}\right) \Gamma\left(t_{1}, y_{1} ; \tau, \xi\right) d y_{1} \cdots d y_{m}
$$

Now we have

$$
t_{i+1}-t_{i}=\frac{t-\tau}{m+1} \leq \frac{T}{m+1} \leq T_{\lambda}, \quad i=0, \ldots, m
$$

by definition of $m$. Moreover, if $y_{i} \in D\left(x_{i} ; r\right)$ for $i=1, \ldots, m$, by the triangular inequality we have

$$
\left|y_{i+1}-y_{i}\right| \leq 2 r+\left|x_{i+1}-x_{i}\right|=2 r+\frac{|x-\xi|}{m+1} \leq
$$

(again, by definition of $m$ )

$$
\leq 2 r+\frac{\varrho_{\lambda}}{2} \sqrt{\frac{t-\tau}{m+1}} \leq \varrho_{\lambda} \sqrt{\frac{t-\tau}{m+1}}
$$

if we set

$$
r=\frac{\varrho_{\lambda}}{4} \sqrt{\frac{t-\tau}{m+1}}>0 .
$$

For such a choice of $r$, we can use (4.21) repeatedly in (4.22) and get

$$
\begin{gathered}
\Gamma(t, x ; \tau, \xi) \geq\left(2 \lambda^{d}\right)^{-(m+1)} \int_{\mathbb{R}^{m d}} \Gamma^{\frac{1}{\lambda}}\left(\frac{t-\tau}{m+1}, x-y_{m}\right) \mathbb{1}_{D\left(x_{m} ; r\right)}\left(y_{m}\right) \prod_{i=1}^{m-1} \Gamma^{\frac{1}{\lambda}}\left(\frac{t-\tau}{m+1}, y_{i+1}-y_{i}\right) \times \\
\times \mathbb{1}_{D\left(x_{i} ; r\right)}\left(y_{i}\right) \Gamma^{\frac{1}{\lambda}}\left(\frac{t-\tau}{m+1}, y_{1}-\xi\right) d y_{1} \cdots d y_{m}
\end{gathered}
$$

(by (4.23) and denoting by $\omega_{d}$ the volume of the unit ball in $\mathbb{R}^{d}$ )

$$
\geq\left(2 \lambda^{d}\right)^{-(m+1)}\left(\omega_{d} r^{d}\right)^{m}\left(\frac{\lambda(m+1)}{2 \pi(t-\tau)}\right)^{\frac{d}{2}(m+1)} \exp \left(-\frac{\lambda \varrho_{\lambda}^{2}}{2}(m+1)\right) .
$$

It follows that there exists a positive constant $C=C(\lambda, \alpha, d, T)$ such that

$$
\Gamma(t, x ; \tau, \xi) \geq \frac{1}{C(t-\tau)^{\frac{d}{2}}} \exp (-C m),
$$

and this implies the required estimate. 


\subsection{Proof of Theorem 2.5}

For any fixed $\tau \in[0, T)$, we consider the stochastic flow $X_{\tau, t}$ defined as in (2.2) for $t \in[\tau, T]$. Let $L_{t}^{(\tau)}$ be the operator defined as in (3.12)-(3.13) through the random change of variable $X_{\tau, t}$. By Theorem 3.4 $L_{t}^{(\tau)}$ is a parabolic operator on the strip $[\tau, T] \times \mathbb{R}^{d}$ with random coefficients, that satisfies Assumptions 4.1 and 4.2 for almost every $\omega \in \Omega$. Then, by Theorem 4.6, $L_{t}^{(\tau)}$ admits a fundamental solution $\Gamma^{(\tau)}(t, x ; \tau, \xi)$ defined for $t \in(\tau, T]$ and $x, \xi \in \mathbb{R}^{d}$. We put

$$
\boldsymbol{\Gamma}(t, x ; \tau, \xi):=\Gamma^{(\tau)}\left(t, X_{\tau, t}^{-1}(x) ; \tau, \xi\right), \quad t \in(\tau, T], x, \xi \in \mathbb{R}^{d} .
$$

Combining Theorems 3.4 and 4.6 we infer that $\boldsymbol{\Gamma}(\cdot, \cdot ; \tau, \xi) \in \mathbf{C}_{\tau, T}^{2}\left(\mathbb{R}^{d}\right)$ and satisfies (2.1) with probability one. Moreover, let us consider a continuous and non-rapidly increasing function $\varphi$ on $\mathbb{R}^{d}$; proceeding as in the proof of Theorem 4.6 we have

$$
\int_{\mathbb{R}^{d}} \boldsymbol{\Gamma}(t, x ; \tau, y) \varphi(y) d y-\varphi(\xi)=I_{1}(t, x, \tau)+I_{2}(t, x, \tau)
$$

where $I_{2}(t, x, \tau)$ is defined and can be estimated as in (4.19); whereas, recalling the definition of parametrix in (4.12) (4.13), we have

$$
\begin{aligned}
\lim _{\substack{(t, x) \rightarrow(\tau, \xi) \\
t>\tau}} I_{1}(t, x, \tau) & =\lim _{\substack{(t, x) \rightarrow(\tau, \xi) \\
t>\tau}} \int_{\mathbb{R}^{d}} \Gamma^{\text {heat }}\left(A_{\tau, t}(y), X_{\tau, t}^{-1}(x)-y\right)(\varphi(y)-\varphi(\xi)) d y \\
& =\lim _{\substack{(t, x) \rightarrow(\tau, \xi) \\
t>\tau}} \int_{\mathbb{R}^{d}} \Gamma^{\text {heat }}\left(A_{\tau, t}\left(X_{\tau, t}^{-1}(x)-y\right), y\right)\left(\varphi\left(X_{\tau, t}^{-1}(x)-y\right)-\varphi(\xi)\right) d y=0
\end{aligned}
$$

by the dominated convergence theorem. This proves that $\boldsymbol{\Gamma}$ is a fundamental solution for the SPDE (1.2).

The Gaussian bounds (2.5) follow directly from the definition (4.24) and the analogous estimates (4.9) for $\Gamma^{(\tau)}$ in Theorem 4.7 Moreover, since

$$
\partial_{x_{i}} \boldsymbol{\Gamma}(t, x ; \tau, \xi)=\left(\nabla \Gamma^{(\tau)}\right)\left(t, X_{\tau, t}^{-1}(x) ; \tau, \xi\right) \partial_{i} X_{\tau, t}^{-1}(x),
$$

the gradient estimate (2.6) follows from the analogous estimate (4.10) for $\Gamma^{(\tau)}$ and from Proposition 3.2. The proof of (2.7) is analogous.

Remark 4.11. Assumption 2.4 is crucial in that it allows us to prove that $\nabla X_{t}(x)$ in (3.7) is finite P-almost surely. In turn, this guarantees that the PDE obtained through the Itô-Wentzell change of coordinates is uniformly parabolic. It would be interesting to investigate to what extent it can be relaxed and if it is possible to have some explicit estimate.

Remark 4.12. More general SPDEs of the form

$$
d v_{t}(x)=\left(\mathbf{L}_{t} v_{t}(x)+\mathbf{f}_{t}(x)\right) d t+\left(\mathbf{G}_{\sigma_{t}^{k}} v_{t}(x)+\mathbf{h}_{t}^{k}(x) v_{t}(x)+\mathbf{g}_{t}^{k}(x)\right) d W_{t}^{k},
$$

can be included in our analysis. Following the argument in [15], proof of Theor.2.7, the idea would be to consider the transformation

$$
v_{t}(x)=\mathcal{E}_{t}(x)\left(u_{t}(x)+\int_{0}^{t} \mathcal{E}_{s}^{-1}(x) \mathbf{g}_{s}^{k}(x) d W_{s}^{k}\right),
$$

where

$$
\mathcal{E}_{t}(x)=\exp \left(\int_{0}^{t} \mathbf{h}_{s}^{k}(x) d W_{s}^{k}-\frac{1}{2} \int_{0}^{t}\left|\mathbf{h}_{s}^{k}(x)\right|^{2} d s\right) .
$$

In fact, if $u$ solves (1.2) then by the Itô formula we have

$$
\begin{aligned}
d v_{t}(x)= & \left(\mathcal{E}_{t}(x) \mathbf{L}_{t}\left(\mathcal{E}_{t}^{-1}(x) v_{t}(x)\right)+\mathcal{E}_{t}(x) \mathbf{f}_{t}(x)\right) d t \\
& +\left(\mathcal{E}_{t}(x) \mathbf{G}_{\sigma_{t}^{k}}\left(\mathcal{E}_{t}^{-1}(x) v_{t}(x)\right)+\mathbf{h}_{t}^{k}(x) v_{t}(x)+\mathbf{g}_{t}^{k}(x)\right) d W_{t}^{k} .
\end{aligned}
$$




\section{References}

[1] Chow, P. L., And Jiang, J.-L. Stochastic partial differential equations in Hölder spaces. Probab. Theory Related Fields 99, 1 (1994), 1-27.

[2] Deck, T., And Kruse, S. Parabolic differential equations with unbounded coefficients - a generalization of the parametrix method. Acta Appl. Math. 74, 1 (2002), 71-91.

[3] Denis, L., Matoussi, A., And Stoica, L. $L^{p}$ estimates for the uniform norm of solutions of quasilinear SPDE's. Probab. Theory Related Fields 133, 4 (2005), 437-463.

[4] Di Francesco, M., and Pascucci, A. On a class of degenerate parabolic equations of Kolmogorov type. AMRX Appl. Math. Res. Express 3 (2005), 77-116.

[5] Fabes, E. B., And Stroock, D. W. A new proof of Moser's parabolic Harnack inequality using the old ideas of Nash. Arch. Rational Mech. Anal. 96, 4 (1986), 327-338.

[6] Flandoli, F. Dirichlet boundary value problem for stochastic parabolic equations: compatibility relations and regularity of solutions. Stochastics Stochastics Rep. 29, 3 (1990), 331-357.

[7] Friedman, A. Partial differential equations of parabolic type. Prentice-Hall Inc., Englewood Cliffs, N.J., 1964.

[8] Fujisaki, M., Kallianpur, G., and Kunita, H. Stochastic differential equations for the non linear filtering problem. Osaka J. Math. 9 (1972), 19-40.

[9] Il' in, A. M., Kalahsnikov, A. S., and Oleinik, O. A. Second-order linear equations of parabolic type. Uspehi Mat. Nauk 17, 3 (105) (1962), 3-146.

[10] Konakov, V., Menozzi, S., And Molchanov, S. Explicit parametrix and local limit theorems for some degenerate diffusion processes. Ann. Inst. Henri Poincaré Probab. Stat. 46, 4 (2010), $908-923$.

[11] KRYLOv, N. V. On $L_{p}$-theory of stochastic partial differential equations in the whole space. SIAM J. Math. Anal. 27, 2 (1996), 313-340.

[12] Krylov, N. V. An analytic approach to SPDEs. In Stochastic partial differential equations: six perspectives, vol. 64 of Math. Surveys Monogr. Amer. Math. Soc., Providence, RI, 1999, pp. 185242.

[13] KRYLOV, N. V. Hörmander's theorem for parabolic equations with coefficients measurable in the time variable. SIAM J. Math. Anal. 46, 1 (2014), 854-870.

[14] Krylov, N. V. Hörmander's theorem for stochastic partial differential equations. Algebra i Analiz 27, 3 (2015), 157-182.

[15] KRYLOV, N. V. Hypoellipticity for filtering problems of partially observable diffusion processes. Probab. Theory Related Fields 161, 3-4 (2015), 687-718.

[16] Krylov, N. V., And Rozovskit, B. L. The Cauchy problem for linear stochastic partial differential equations. Izv. Akad. Nauk SSSR Ser. Mat. 41, 6 (1977), 1329-1347, 1448. 
[17] Kunita, H. Stochastic flows and stochastic differential equations, vol. 24 of Cambridge Studies in Advanced Mathematics. Cambridge University Press, Cambridge, 1990.

[18] Mikulevicius, R. On the Cauchy problem for parabolic SPDEs in Hölder classes. Ann. Probab. 28, 1 (2000), 74-103.

[19] Pagliarani, S., Pascucci, A., And Pignotti, M. Intrinsic expansions for averaged diffusion processes. Stochastic Process. Appl. 127, 8 (2017), 2560-2585.

[20] Pardoux, E. Équations aux dérivées partielles stochastiques de type monotone. Séminaire sur les Équations aux Dérivées Partielles (1974-1975), III, Exp. No. 2 (1975), 10.

[21] Pascucci, A., And Pesce, A. On stochastic Langevin and Fokker-Planck equations: the twodimensional case. arXiv:1910.05301v1 (2019).

[22] Polidoro, S. On a class of ultraparabolic operators of Kolmogorov-Fokker-Planck type. Matematiche (Catania) 49, 1 (1994), 53-105.

[23] QIU, J. Hörmander-type theorem for Itô processes and related backward SPDEs. Bernoulli 24, 2 (2018), 956-970.

[24] Rozovskit, B. L. Stochastic partial differential equations. Mat. Sb. (N.S.) 96(138) (1975), 314341,344 .

[25] Rozovski, B. L. Stochastic evolution systems, vol. 35 of Mathematics and its Applications (Soviet Series). Kluwer Academic Publishers Group, Dordrecht, 1990. Linear theory and applications to nonlinear filtering, Translated from the Russian by A. Yarkho.

[26] Shimizu, A. Fundamental solutions of stochastic partial differential equations arising in nonlinear filtering theory. In Probability theory and mathematical statistics (Tbilisi, 1982), vol. 1021 of Lecture Notes in Math. Springer, Berlin, 1983, pp. 594-602.

[27] Sowers, R. B. Recent results on the short-time geometry of random heat kernels. Math. Res. Lett. 1, 6 (1994), 663-675.

[28] Sowers, R. B. Short-time geometry of random heat kernels. Mem. Amer. Math. Soc. 132, 629 (1998), viii+130.

[29] Ventcel, A. D. On equations of the theory of conditional Markov processes. Theory Probability and Appl. 10 (1965), 357-361.

[30] Zatezalo, A. Filtering of partially observable stochastic processes. ProQuest LLC, Ann Arbor, MI, 1998. Thesis (Ph.D.)-University of Minnesota. 